\section{Automatic Application of Mobile Phase for Descending Paper Chromatography}

\author{
D. M. ABELSON AND J. G. C. FOX \\ From the Departments of Medicine and of Biophysics, \\ Postgraduate Medical School of London
}

(RECEIVED FOR PUBLICATION AUGUST 22, 1958)

When volatile solvents are used for paper chromatography, as in the systems described by Bush (1952) for separation of steroids, it is usually necessary to hang the paper in the tank for several hours' equilibration before the addition of the mobile phase. If equilibration is allowed to proceed overnight much of the next day will be taken up with running the chromatogram. An apparatus has therefore been constructed which delivers the mobile phase into the upper trough at a pre-arranged time during the night, so that development of the chromatogram is completed by morning.

The mobile phase is contained in a cylindrical glass chamber (Fig. 1) constructed from a $250 \mathrm{ml}$. beaker or suitable length of wide-bore tubing. The chamber rests on the glass plate forming the roof of the chromatography tank, and is provided with (1) a filling tube, normally closed by a ground-glass stopper, and (2) a horizontal outlet tube, inserted a little above the floor of the chamber, so that any stationary phase which may have been added will not be carried over into the trough. This tube contains a $4 \mathrm{~mm}$. two-way spring-loaded stopcock. Beyond the stopsock the tube turns downwards and passes through the roof of a small glass dome covering a

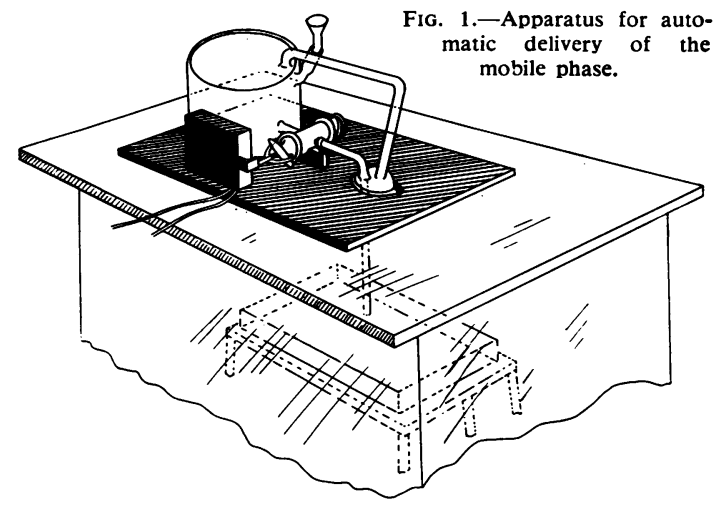

hole in the glass plate, and thence into the upper trough of the chromatography tank. The base of the dome is ground to form an airtight seal with the glass plate.

The handle of the tap is embraced by two curved metal arms, soldered to the spindle of a solenoidactuated rotary switch. The spindle rotates through $60^{\circ}$ when the solenoid is energized. The switch is connected via a "sangamo" 24-hour mains-operated time clock (with separate motor connexions and a day-omitting device) to a 12-volt car battery (Fig. 2). By careful adjustment of the contact arms of the time clock the "on" time can be reduced to 50-70 seconds. As an additional precaution, and to prevent the solenoid heating up unduly, a 12-volt "flasher" (Bulgin thermal delay switch Type S586) is incorporated in the circuit to disconnect the solenoid about seven seconds after the current has been turned on by the time clock. Flasher, battery, and time clock are situated some distance from the apparatus, which can therefore be placed in position inside a thermostatically controlled heating box without danger of sparking in the inflammable atmosphere of the volatile solvents.

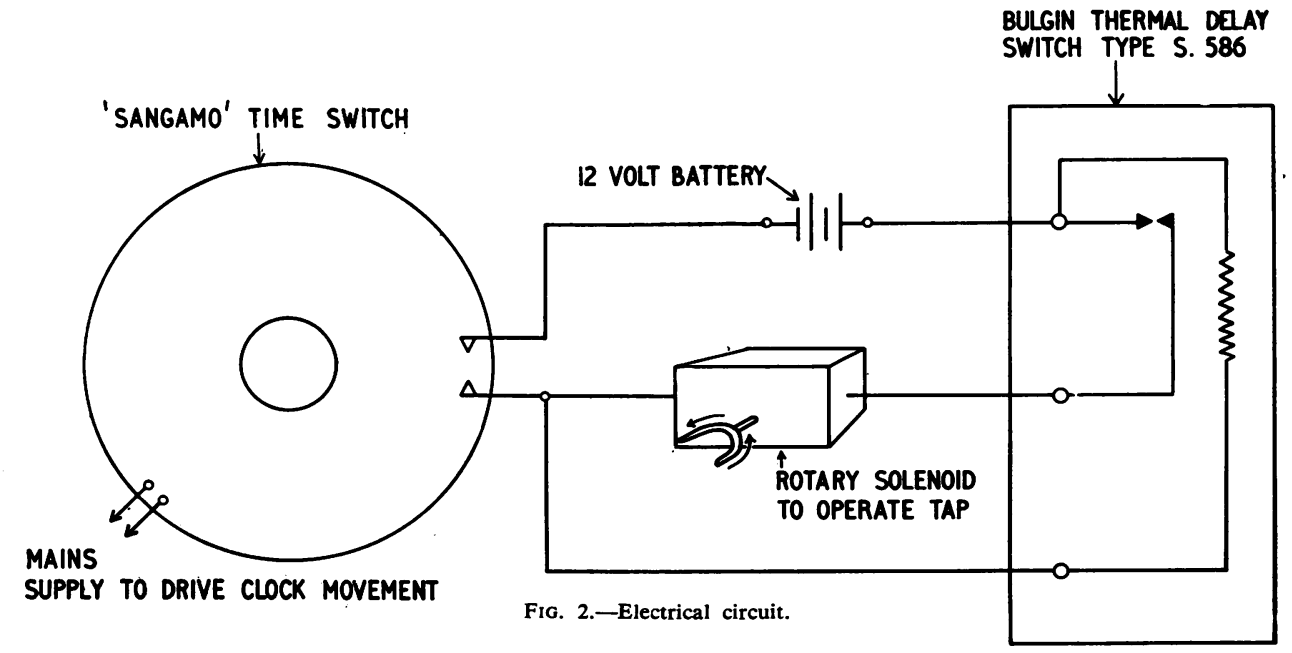

2L 
(3) A return tube from the glass dome leads back to the top of the chamber. Air displaced from the chromatography tank by the incoming fluid escapes through the hole in the glass plate into the dome and passes via the return tube to the chamber, thus forming a completely closed system.

Added rigidity is afforded by mounting the apparatus on a "tufnol" plate, to which the glass chamber, stopcock, and rotary switch are firmly secured.

We are indebted to the University of London for a grant for apparatus.

REFERENCE

Bush, I. E. (1952). Biochem. J., 50, 370.

\section{Practical Laboratory Tests for the Identification} of Proteus Strains

\section{U. BACHRACH AND M. WORMSER}

From the Department of Clinical Microbiology, Hebrew University-Hadassah Medical School, Jerusalem, Israel

(RECEIVED FOR PUBLICATION AUGUST 6, 1958)

The rapid identification of Proteus strains is of importance since these relatively non-pathogenic micro-organisms are easily confused with other lactose - negative, pathogenic bacteria such as Salmonellae.

In many laboratories Proteus species are identified by their urease activity, usually by inoculating bacterial cells into a medium containing urea plus an indicator and noting the colour change after four to eight hours (Rustigian and Stuart, 1943). Since urea is decomposed by autoclaving, the medium must be sterilized by Seitz filtration. This complicates the technique and increases the possibility of contamination.

Proom and Woiwod (1951) proposed the use of a method based on the ability of certain Proteus species to form volatile amines. The procedure, however, is rather complicated and could hardly be used for routine diagnostic purposes.

More recently Singer and Volcani (1955) have described a simple and rapid method, based on the decomposition of tryptophan by Proteus cells to form a compound giving a brown colour with ferric chloride.

The present paper describes two modified techniques, one for detecting urease activity, and the other a simple spot test for the detection of volatile amines. The two modified methods are compared with the tryptophan-ferric chloride test of Singer and Volcani (1955).

\section{Reagents}

Procedure A.- The following are required:

Urea Solution.-Dissolve $1.0 \mathrm{~g}$. urea and $0.1 \mathrm{~g}$. phenolphthalein in $10 \mathrm{ml} .96 \%$ ethyl alcohol.

Saline Solution.-Make up $8.5 \mathrm{~g}$. sodium chloride in $100 \mathrm{ml}$. distilled water.

Dilute Hydrochloric Acid Solution.-Add $1 \mathrm{ml}$. concentrated hydrochloric acid to $100 \mathrm{ml}$. distilled water.

Sodium Hydroxide Solution.-Add 0.4 g. sodium hydroxide to $100 \mathrm{ml}$. distilled water.

Procedure B.-The following are required:

2: 4 Dinitro-fluoro-benzene Reagent (D.N.F.B.).Add $0.65 \mathrm{ml}$. dinitro-fluoro-benzene to $50 \mathrm{ml}$. acetone.

Potassium Hydroxide Solution.-Dissolve $40 \mathrm{~g}$. potassium hydroxide in $100 \mathrm{ml}$. distilled water. 